\title{
Closing In on Mid-IR Pulsed All-Fiber Lasers Based on Saturable Absorbers
}

\author{
Pascal Paradis ${ }^{1}$, Vincent Fortin ${ }^{1}$, Bernard Dussardier ${ }^{2}$, Réal Vallée ${ }^{1}$, Martin \\ Bernier ${ }^{1}$ \\ 1. Centre d'optique, photonique et laser (COPL), Université Laval, Québec City, Québec G1V 0A6, Canada \\ 2. Institut de Physique de Nice, Université Côte d'Azur, CNRS, 06108 Nice CEDEX 2, France \\ pascal.paradis.2@ulaval.ca
}

\begin{abstract}
Novel schemes are proposed for building an all-fiber mode-locked laser cavity operating at 2.8 microns based on a heavily erbium-doped fluoride fiber bounded by a semiconductor saturable absorber mirror and a fiber Bragg grating. ๑ 2020 The Author(s)
\end{abstract}

\section{Introduction}

Mid-infrared (mid-IR) pulsed fiber lasers are promising for laser surgery due to the strong water absorption peak at $2.94 \mu \mathrm{m}$ [1]. These lasers would also be advantageous for field applications such as remote sensing because their emission spectrum can reach the fundamental rotation-vibrational resonances of many atmospheric pollutants such as methane [2]. In recent years, a multitude of ultrafast fiber lasers operating near $3 \mu \mathrm{m}$ have been reported. Ring cavity lasers using nonlinear polarization rotation evolution generated pulses as short as $180 \mathrm{fs}$ [3], while those using a saturable absorber (SA) emitted pulses as short as 6 ps [4]. A simpler linear mode-locked fiber laser based on a semiconductor saturable absorber mirror (SESAM) and a fiber Bragg grating (FBG) was also reported, producing 60 ps pulses limited by the narrow FBG bandwidth [5]. A more traditional mode-locked fiber laser based on a SESAM and a broadband output coupler generated 25 ps pulses [6]. However, the building cost and alignment requirements of these lasers restrict their field deployment and limit their usage by laser experts.

In this paper, we present a mode-locked erbium-doped fluoride fiber laser, based on an FBG and a SESAM, generating 15 ps pulses at $2.825 \mu \mathrm{m}$ with $150 \mathrm{~mW}$ average output power at a repetition rate of $55 \mathrm{MHz}$ as a step towards ultrafast all-fiber lasers emitting in the mid-IR.

\section{Experimental Setup}

The laser setup, as illustrated in Fig. 1(a), is made of a $2 \mathrm{~m}$-long $7 \% \mathrm{~mol} \mathrm{Er}^{3+}$-doped fluoride fiber (Le Verre Fluoré) as the gain medium for the laser cavity bounded by a SESAM (BATOP GmbH) and an FBG written through the fiber's protective coating using femtosecond pulses to preserve its original robustness [7]. The gain fiber is backward pumped with a $976 \mathrm{~nm}$ pump diode (Alfalight, B33125) to simplify the intracavity alignment and achieve higher output power than in a forward-pumping scheme. The FBG has a maximum reflectivity of $65 \%$ at $2790.9 \mathrm{~nm}$ with a FWHM of $3.2 \mathrm{~nm}$. The aspherical ZnSe lenses have focal lengths of $25 \mathrm{~mm}$ at the output and $12.5 \mathrm{~mm}$ inside the cavity, and each of them transmits around $95 \%$ of the signal at $2.8 \mu \mathrm{m}$. The fiber tips are angle-cleaved at $8^{\circ}$ to eliminate parasitic reflections thus achieving stable mode-locking and the residual pump power is removed by the dichroic mirror (DM) to avoid damaging the SESAM. Moreover, we propose the use of a fiber-based saturable absorber mirror (SAM) and a pump combiner to achieve a robust pulsed all-fiber linear laser emitting around $2.8 \mu \mathrm{m}$ as shown in Fig. 1(b).

\section{Results}

This mode-locked laser cavity generates 15 ps pulses with a time-bandwidth product of 0.380 , i.e., close to the Fourier-Transform limit as shown in Fig. 2. The mode-locked regime is self-starting without any Q-switched regime at lower pump power if the alignment of the intracavity components is optimal. The mode-locked regime is stable with one pulse per round trip between $87.5 \mathrm{~mW}$ and $157.5 \mathrm{~mW}$ of average output power. At higher power, the mode-locked regime becomes unstable with two pulses per round trip with longer pulse duration. Moreover, the emission spectrum is centered on the FBG's minimum transmission (i.e., on its maximum reflectivity) which indicates that the duration and the spectrum can be controlled by the FBG's properties in this design if the total cavity dispersion is low enough in the anomalous regime and the SA's recovery time is short enough, its modulation depth is not too shallow and the saturation fluence is high enough. 


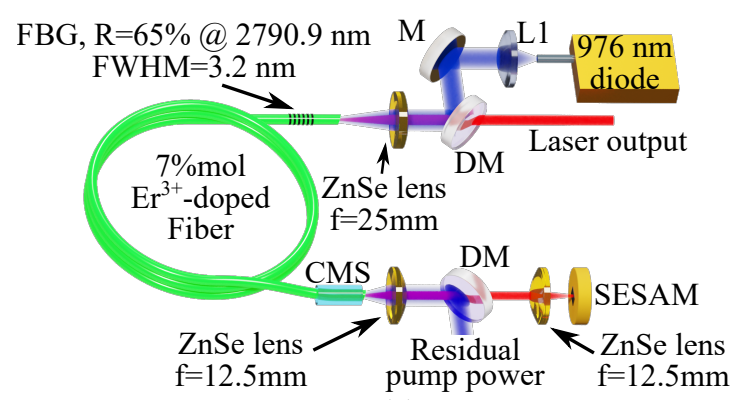

(a)

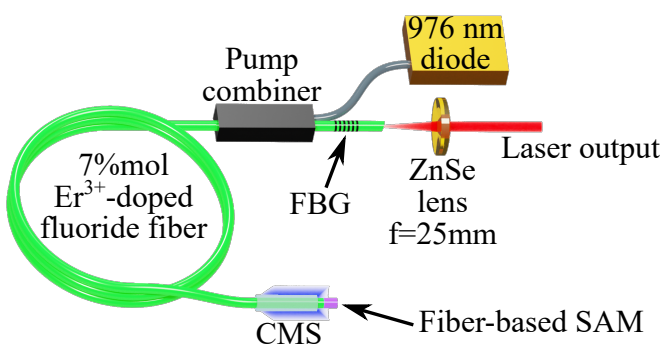

(b)

Fig. 1. a) Experimental setup of the mode-locked $\mathrm{Er}^{3+}$-doped fiber laser based on a SESAM and an FBG. b) Proposed setup for the mode-locked $\mathrm{Er}^{3+}$-doped fiber laser based on a SA fiber and an FBG. (CMS: cladding mode stripper, DM: dichroic mirror, M: gold mirror, L1: silica lens $\mathrm{f}=11 \mathrm{~mm}$ )

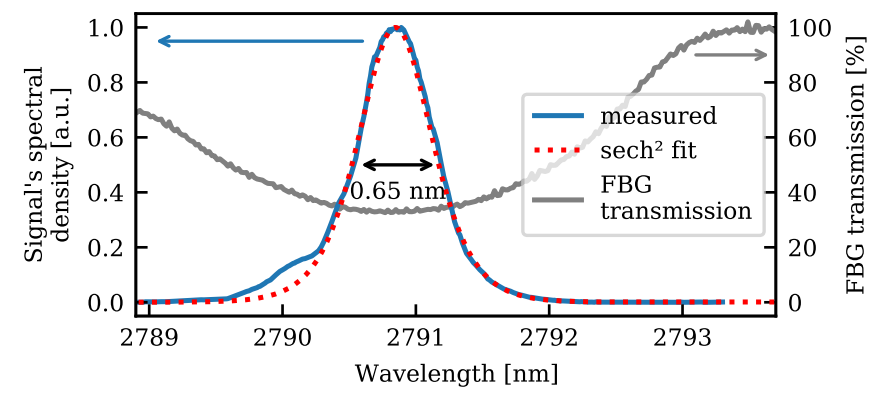

(a)

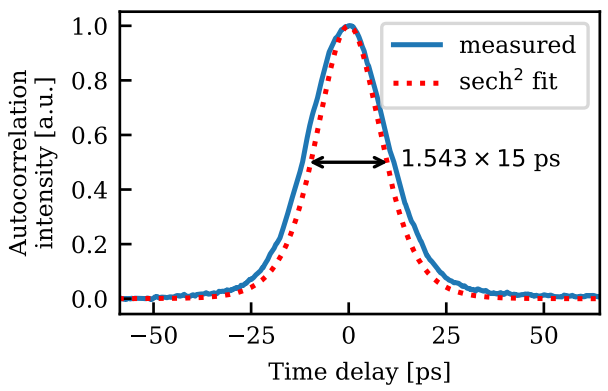

(b)

Fig. 2. a) Experimental signal's spectral density and FBG's transmission spectrum and b) duration of the pulses generated by the laser source

\section{Conclusion}

We have presented a simple mode-locked fiber laser design based on a SESAM and an FBG that allows control of the duration and the bandwidth of the pulses. While being much simpler than ultrafast ring lasers operating in Mid-IR with a saturable absorber, this laser design was able to generate 15 ps pulses close to the Fourier-transform limit. During the presentation, different new configurations of fiber-based SA mirror will be discussed to greatly improve the robustness and reliability of ultrafast mid-IR lasers.

\section{References}

1. X. Zhu, G. Zhu, C. Wei, L. V. Kotov, J. Wang, M. Tong, R. A. Norwood, and N. Peyghambarian, "Pulsed fluoride fiber lasers at $3 \mu \mathrm{m}$ [Invited]," J. Opt. Soc. Am. B 34, A15 (2017).

2. F. K. Tittel, D. Richter, and A. Fried, "Mid-Infrared Laser Applications in Spectroscopy," in Solid-State Mid-Infrared Laser Sources, (Springer Berlin Heidelberg, Berlin, Heidelberg, 2003), pp. 458-529.

3. S. Antipov, D. D. Hudson, A. Fuerbach, and S. D. Jackson, "High-power mid-infrared femtosecond fiber laser in the water vapor transmission window," Optica 3, 1373 (2016).

4. T. Hu, D. D. Hudson, and S. D. Jackson, "Stable, self-starting, passively mode-locked fiber ring laser of the $3 \mu \mathrm{m}$ class," Opt. Lett. 39, 2133 (2014).

5. A. Haboucha, V. Fortin, M. Bernier, J. Genest, Y. Messaddeq, and R. Vallée, "Fiber Bragg grating stabilization of a passively mode-locked $28 \mu \mathrm{m} \mathrm{Er}^{3+}$ : fluoride glass fiber laser," Opt. Lett. 39, 3294 (2014).

6. P. Tang, Z. Qin, J. Liu, C. Zhao, G. Xie, S. Wen, and L. Qian, "Watt-level passively mode-locked Er ${ }^{3+}$-doped ZBLAN fiber laser at $28 \mu \mathrm{m}$,’ Opt. Lett. 40, 4855 (2015).

7. J. Habel, T. Boilard, J.-S. Frenière, F. Trépanier, and M. Bernier, "Femtosecond FBG Written through the Coating for Sensing Applications," Sensors 17, 2519 (2017). 